\title{
Responsible use of rifampin for the treatment of latent tuberculosis infection
}

\author{
Jane Batt MD PhD, Kamran Khan MD MPH
}

Cite as: CMAJ 2019 June 24;191:E678-9. doi: 10.1503/cmaj.190081

$\mathbf{T}$ he current standard of care for treatment of latent tuberculosis infection (LTBI), as specified by the Canadian Tuberculosis Standards, ${ }^{1}$ is 9 months of daily isoniazid. A recent landmark clinical trial published by Menzies and colleagues, involving more than 6000 patients across 9 countries, showed that 4 months of rifampin is noninferior to, better tolerated than and has higher treatment completion rates than 9 months of isoniazid for the prevention of active tuberculosis (TB) among patients with LTBI. ${ }^{2}$ These compelling findings will undoubtedly expand the use of rifampin for the treatment of LTBI. Although treatment of LTBI is a critical component of efforts to eliminate TB in high-income countries and of the World Health Organization's (WHO) End TB Strategy (www.who.int/tb/ strategy/end-tb/en/), ${ }^{3}$ there are important risks that must be considered. Specifically, use of rifampin monotherapy to treat individuals who are presumed to have LTBI but who actually have subclinical active TB could induce the development of rifampin resistance, with serious consequences to the individual and society.

Before beginning treatment for LTBI with either rifampin or isoniazid, the treating clinician must rule out active $\mathrm{TB}^{1}$ because the Mycobacterium tuberculosis bacterium can rapidly develop resistance to a single antimicrobial (https://apps.who.int/iris/ bitstream/handle/10665/42701/9241546034.pdf). ${ }^{4}$ To rule out active TB with confidence, patients must be assessed for recent exposures and compatible symptoms, undergo a thorough physical examination and have a chest $\mathrm{x}$-ray to look for radiographic signs of TB. ${ }^{4}$ Selected patients with TB risk factors or clinical findings of TB or both must also have sputum collected for TB smear and culture, ${ }^{4}$ which, in some situations, will include individuals who have a normal physical examination, a normal chest radiograph and no symptoms of active TB. For example, close contacts of a patient with active TB may develop primary disease in the absence of any clinical or radiographic findings, and as many as $70 \%$ of HIV-positive individuals with asymptomatic pulmonary TB will have normal chest $\mathrm{x}$-rays. ${ }^{5}$ Sputum collection is therefore required to avoid missing subclinical active disease.

Similarly, among patients with chest radiographic findings consistent with TB, such as upper-lobe fibronodular changes, sputum must be collected to rule out active TB. The Canadian TB

\section{KEY POINTS}

- A regimen of daily rifampin for 4 months for people with latent tuberculosis infection (LTBI) has recently been shown to have higher treatment completion rates, a more favourable adverse effect profile and effectiveness comparable to a 9-month regimen of daily isoniazid.

- Before beginning treatment for LTBI with rifampin monotherapy, clinicians must confidently rule out active TB using a thorough clinical and radiographic examination and, in some cases, sputum cultures - to prevent the inadvertent development of rifampin resistance.

- As rifampin is the most important drug for the treatment of active TB, the development of rifampin resistance carries serious consequences to patients and society.

Standards recommend that 3 sputum specimens be obtained, to increase sensitivity of testing; whereas 1 sputum specimen is between $54 \%$ and $80 \%$ sensitive in detecting TB in culture, additional samples provide incremental improvement, with the yield of the third as high as an additional $5 \%$ to $10 \% .{ }^{4,6}$ Because spontaneous sputum specimens are often difficult to obtain in patients without respiratory symptoms, sputum induction may be necessary. ${ }^{1,7}$ However, in solo or small group practices, especially in remote communities, sputum induction may not be possible, which may complicate the clinician's ability to rule out active TB before safely beginning treatment for LTBI.

In the landmark rifampin study, trial sites were in settings that had widely varying levels of resources. ${ }^{2}$ Regardless, before therapy for LTBI was started, sputum samples for TB smear and culture were required, to rule out active disease in individuals with chest $\mathrm{x}$-ray abnormalities compatible with TB (lesion $>2 \mathrm{~cm}^{2}$ ), or close contacts with symptoms or chest $x$-ray abnormalities consistent with active TB. ${ }^{2}$ Compared with isoniazid, rifampin was better tolerated $(5.8 \%$ v. $2.8 \%$ adverse reactions, respectively) and had higher treatment completion rates $(63.2 \%$ v. $78.8 \%$, respectively). In the 28 -month follow-up period, there were 8 cases of confirmed active TB: 4 with rifampin and 4 with isoniazid. One patient developed isoniazid-resistant TB 2 months after starting isoniazid for LTBI, and a second developed active 
TB less than 2 months after completing a 4-month course of rifampin. The isolate from the latter individual showed rifampin resistance on genotypic testing but the bacterium was fully sensitive with phenotyping testing. It is important to note, however, that HIV and other immunosuppressed populations were not well represented in the study $(4.0 \%$ and $3.2 \%$, respectively); nor were individuals with fibronodular disease on chest radiographs (5.0\%); most patients were close contacts of patients with active TB $(70.7 \%)$ and $78.4 \%$ had normal chest radiographs. We believe that this study cohort does not adequately reflect the mix of patients that Canadian clinicians encounter in practice (e.g., newly arriving immigrants with upper-lobe fibronodular disease on chest imaging).

It is important to note that rifampin and isoniazid resistance have very different clinical implications. Rifampin is unequivocally the most important drug in the global fight against TB. The loss of isoniazid in the management of active TB is undesirable, but it does not lengthen or complicate the standard 6-month treatment regimen because other drugs (i.e., ethambutol or fluoroquinolones or both) can be used in its place. However, if rifampin cannot be used, the Canadian TB standards recommends extension of TB treatment to 1 year with the remaining first-line drugs and an injectable agent (e.g., aminoglycoside), and in the absence of the injectable drug, a minimum of 18 months of oral treatment. ${ }^{4}$ The WHO recently recommended that rifampin monoresistance be treated as multidrug-resistant TB (resistant to at least rifampin and isoniazid), ${ }^{8}$ meaning a 9- to 20-month treatment regimen with 5 to 7 second-line TB antibiotics, including an injectable drug, which is associated with substantial toxicities and increased cost. ${ }^{9}$ Prolongation of therapy also adversely affects patient productivity. ${ }^{10}$ Furthermore, the inadvertent use of rifampin for treatment of $\mathrm{LTBI}$ in an individual with subclinical isoniazid-resistant TB (about $8 \%$ of Canadian TB isolates are resistant to isoniazid) could lead to multidrug-resistant TB.

It is noteworthy that the development of drug resistance has not been frequently observed in other clinical trials of therapy for LTBI. ${ }^{11,12}$ A recent systematic review and meta-analysis of 6 randomized clinical trials looking at rifampin resistance with LTBI therapy found just 6 cases of resistance among 6808 individuals treated for LTBI with a rifamycin-containing regimen. It is also noteworthy that of the 6 trials, 5 used a rifamycin in combination with isoniazid or pyrazinamide or both. This highlights that combination drug regimens for $\mathrm{LTBI}$, such as once-weekly isoniazid plus rifapentine for 12 weeks, ${ }^{13}$ could reduce the risk of acquired resistance relative to single drug regimens.

Menzies and colleagues' trial ${ }^{2}$ is an important contribution to our understanding of how to optimize therapy for LTBI and could have profound implications for TB prevention, including in resource-limited environments. Given the tremendous importance of rifampin in the treatment of active TB, clinicians will need to be extremely vigilant in ruling out active TB before prescribing rifampin monotherapy to their patients. Clinicians who lack access to key diagnostic services such as sputum induction should consider referring patients to centres that are experienced in the management of TB.

\section{References}

1. Menzies D, Alvarez GG, Khan K. Treatment of LTBI. In: Canadian tuberculosis standards, 7th ed. Ottawa; Public Health Agency of Canada, The Lung Association, Thoracic Society; 2014.

2. Menzies D, Adjobimey M, Ruslami R, et al. Four months of rifampin or nine months of isoniazid for latent tuberculosis in adults. N Engl J Med 2018;379:440-53.

3. Lönnroth K, Migliori GB, Abubakar I, et al. Towards tuberculosis elimination: an action framework for low-incidence countries. Eur Respir J 2015;45:928-52.

4. Menzies D, Elwood K. Treatment of tuberculosis disease. In: Canadian tuberculosis standards, 7th ed. Ottawa; Public Health Agency of Canada, The Lung Association, Thoracic Society; 2014.

5. Oni T, Burke R, Tsekela R, et al. High prevalence of subclinical tuberculosis in HIV-1-infected persons without advanced immunodeficiency: implications for TB screening. Thorax 2011;66:669-73.

6. Mase SR, Ramsay A, Ng V, et al. Yield of serial sputum specimen examinations in the diagnosis of pulmonary tuberculosis: a systematic review. Int J Tuberc Lung Dis 2007;11:485-95.

7. Gonzalez-Angulo Y, Wiysonge CS, Geldenhuys $\mathrm{H}$, et al. Sputum induction for the diagnosis of pulmonary tuberculosis: a systematic review and meta-analysis. Eur J Clin Microbiol Infect Dis 2012;31:1619-30.

8. WHO treatment guidelines for drug-resistant tuberculosis: 2016 update. Geneva: World Health Organization; 2016.

9. Marks SM, Flood J, Seaworth B, et al. Treatment practices, outcomes, and costs of multidrug-resistant and extensively drug-resistant tuberculosis, United States, 2005-2007. Emerg Infect Dis 2014;20:812-21.

10. Kik SV, Olthof SP, de Vries JT, et al. Direct and indirect costs of tuberculosis among immigrant patients in the Netherlands. BMC Public Health 2009;9:283.

11. den Boon S, Matteelli A, Getahun H. Rifampicin resistance after treatment for latent tuberculous infection: a systematic review and meta-analysis. Int J Tuberc Lung Dis 2016;20:1065-71.

12. van Halsema CL, Fielding KL, Chihota VN, et al. Tuberculosis outcomes and drug susceptibility in individuals exposed to isoniazid preventive therapy in a high HIV prevalence setting. AIDS 2010;24:1051-5.

13. Sterling TR, Villarino ME, Borisov AS, et al. Three months of rifapentine and isoniazid for latent tuberculosis infection. N Engl J Med 2011;365:2155-66.

\section{Competing interests: None declared.}

This article has been peer reviewed.

Affiliations: Keenan Research Centre for Biomedical Science (Batt), St. Michael's Hospital; Division of Respirology, Department of Medicine (Batt), University of Toronto; Li Ka Shing Knowledge Institute (Khan), St. Michael's Hospital; Division of Infectious Diseases, Department of Medicine (Khan), University of Toronto, Toronto, Ont.

Contributors: Both authors equally contributed to the conception and design of the work, drafted the manuscript, revised it critically for important intellectual content, gave final approval of the version to be published and agreed to be accountable for all aspects of the work.

Correspondence to: Jane Batt, jane.batt@utoronto.ca 